\title{
Implementation of Convolutional Neural Network with Optimization Techniques for Face Recognition
}

\author{
Roshni S. Khedgaonkar ${ }^{1}$, Kavita R. Singh², Mukesh M. \\ Raghuwanshi ${ }^{3}$ and Pravinkumar M. Sonsare ${ }^{4}$ \\ ${ }^{1}$ Assistant Professor, Computer Technology, Yeshwantrao Chavan College of Engineering, Nagpur, India \\ ${ }^{2}$ Associate Professor, Computer Technology, Yeshwantrao Chavan College of Engineering, Nagpur, India \\ ${ }^{3}$ Professor, Computer Engineering, G.H. Raisoni College of Engineering and Management, Pune, India \\ ${ }^{4}$ Assistant Professor, Computer Science and Engineering, Shri Ramdeobaba College of \\ Engineering and Management, Nagpur, India
}

\section{ABSTRACT}

Face images is the key factor though which an individuals are distinguished. Because of current pandemic impact of COVID-19 the entire universe is drawing closer towards contact less Gadgets where less physical collaboration is required. In view of this, we have proposed the Convolutional Neural Network (CNN) model and applied on various face databases by measuring the behaviour of various optimizers. The experimentation has been performed on various face databases. In particular, AR, LFW, LAG, DFW, UKFace Database, PSD, ASPS. These databases contains the facial images with variations in illumination, expression, various poses of face image, occluded images by face mask, sunglasses, beared, mustache also contains face images of different age group. Furthermore, the proposed CNN model have evaluated on pre and post plastic surgical face images. The working of some of the optimizers listed as, PowerSign, AddSign, RMSprop, Adam are observed on these face images. From the said optimizers, PowerSign and AddSign perform better for sequential data. However, it comes to 2D pictures its performance diminishes to incredible expand. When performed an experimentation with RMSprop achieved better accuracy. Nevertheless, suffers from the local minimum. On a contrary, Adam outperform with respect to CNN model by obtaining local minima in less time. Adam achieves 98\% to 90\% of accuracy for AR to PSD(Plastic surgery face database) and ASPS(American Society of Plastic Surgeon) database.

KEY WORDS: CONVOLUTIONAL NEURAL NETWORK, LOCAL PLASTIC SURGERY, DEEP LEARNING, MACHINE LEARNING, ADAM OPTIMIZER.

\section{INTRODUCTION}

Face recognition (FR) is useful thoroughly in the field of institutes, data security, crime controlled zones. Generally, face recognition system does not need the

\section{ARTICLE INFORMATION}

*Corresponding Author: roshni.k86@gmail.com

Received 19th Oct 2020 Accepted after revision 29th Dec 2020

Print ISSN: 0974-6455 Online ISSN: 2321-4007 CODEN: BBRCBA

Thomson Reuters ISI Web of Science Clarivate Analytics USA and Crossref Indexed Journal

\section{Clarivate
Analytics}

NAAS Journal Score 2020 (4.31)

A Society of Science and Nature Publication,

Bhopal India 2020. All rights reserved.

Online Contents Available at: http//www.bbrc.in/

Doi: $h$ ttp://dx.doi.org/10.21786/bbrc/13.14/68 sense of touch or interaction from human to conduct the process of recognition. It is one of the benefits of face recognition over the other recognition strategies. for example, The flare-up of novel coronavirus 2019 (COVID-19) has prodded the critical advancement of biometric recognition technologies that can dissect and distinguish subjects wearing facial masks, particularly in high security applications. In spite of the fact that, from last endless many years, face recognition is considered as a developed area of computer vision it actually battles with performance issues because of different challenging issues, for example, variety in pose of a face is because of various angle or edge, from which image of the face has been procured. The variation in illumination emerges

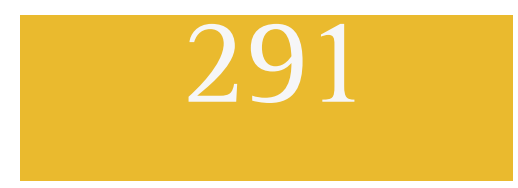


due to uneven distribution of light from different sources on face.

Further, facial images have different expressions that also directly affect the appearance of the facial images. Furthermore, various other obstacles, for example, wearing mask, beard, mustache, and sun glasses, scarf changes the presence of an individual where some segment of face is blocked because of which few feature gets lost (Khadatkar, Khedgaonkar and Patnaik, 2016). Another difficult issue is facial plastic surgery procedure which additionally changes the presence of a face and result in complex issue in face recognition(R.Singh, 2010) Normally, facial plastic surgery is applied for enhancing the facial appearance(Singh, Khedgaonkar and Gawande, 2011). All the aforementioned issues changes the features of face which makes face recognition a challenging task. It may create uncertainty author in(Raghuwanshi and Singh, 2009) handle this through roughest theory.

In earlier study, many researchers have proposed various algorithms for feature extraction listed as, Principal Component Analysis (PCA) (D Xiaoqian , H Huan, 2010), Linear Discriminant Analysis (LDA) (]H Yu, 2001)(]H C Kim , D Kim, 2002)and Local Binary Patterns (LBP)(L Lei, D H Kim, W J Par, 2016). Recently, deep learning based feature extraction algorithm is used which gives incredible advantages (Boureau, Y.-L.; Ponce, J.; LeCun, 2010). Particularly, CNN is the promising technique for face recognition. As per the recent literature, the CNN performed well in an unconstraint environment(Fredj, Hana \&t Bouguezzi, Safa \&t Souani, 2020)(Ridha Ilyas, Bendjillali \&t Beladgham, Moh \& Merit, Khaled \& Miloud, 2020). It worked well with occluded faces The Section II focuses on overview of Convolutional Neural Network. Section III discussed the proposed approach. The detailed database description is given in Section IV. Section V is highlighted the result and conclusion part.

Figure 1. The General Architecture of CNN(Boureau, Y.-L.; Ponce, J.; LeCun, 2010)

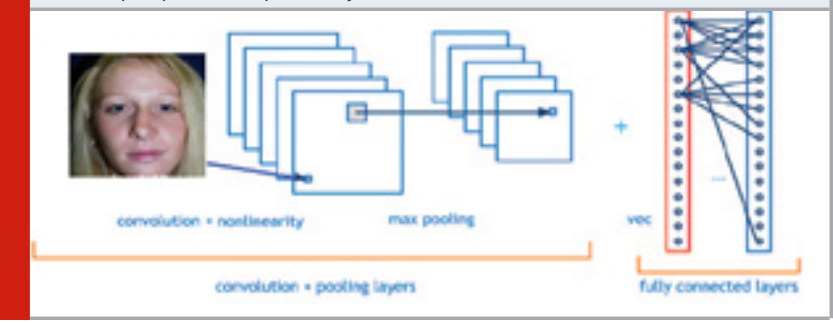

Preliminaries: This section gives the insight of Convolutional Neural network and its various layers as well as the variety of optimizers have been used in this paper. A convolutional neural network is a kind of artificial neural network (ANN). A typical CNN architecture can be seen as shown in Figure1. The structure of CNN contains Convolutional, pooling, Rectified Linear Unit (ReLU), and Fully Connected layers.

Convolutional layer: Convolutional layer is considered as an essential block of CNN. The mathematical representation (Boureau, Y.-L.; Ponce, J.; LeCun, 2010), is represented in Equation (1)

$$
y_{i}=b_{i}+\sum_{x_{i} \in X} w_{i j} * x_{i}
$$

where $\mathrm{y}_{\mathrm{i}} \in \mathrm{Y}, \mathrm{i}=1,2, \ldots \mathrm{D}$. D is the depth of the convolutional layer. Each filter $\mathrm{w}_{\mathrm{ij}}$ is a $3 \mathrm{D}$ matrix of size $\left[\mathrm{F} \times \mathrm{F} \times \mathrm{C}_{-} \mathrm{x}\right]$. Its size is determined by a receptive field $(F)$, and $\left(C_{x}\right)$ is the depth of feature-map. Pooling layers: Pooling layers are mainly authorized for dimensionality reduction which reduces the input dimensions by performing the downsampling operation(Boureau, Y.-L.; Ponce, J.; LeCun, 2010). Fully connected layers: Fully connected (FC) layers transform the two dimensional feature map to the single dimension feature vector. In respect to this, 1D feature vector act as a classifier for classification purpose.

Proposed Approach: Feature extraction is the system of capturing the ideal feature esteems utilizing CNN instead of extraction of hand-crafted features. The general process of face recognition comprises of same traditional steps: Image capture, Feature Extraction and Classification. Features are extracted using CNN instead of hand-crafted way of extraction. The overall process of proposed CNN model consists of various layers as model, 7-layer CNN architecture has designed. The person identification is done be describe in figure 2 .

Figure2: Proposed 7- Layers CNN Model

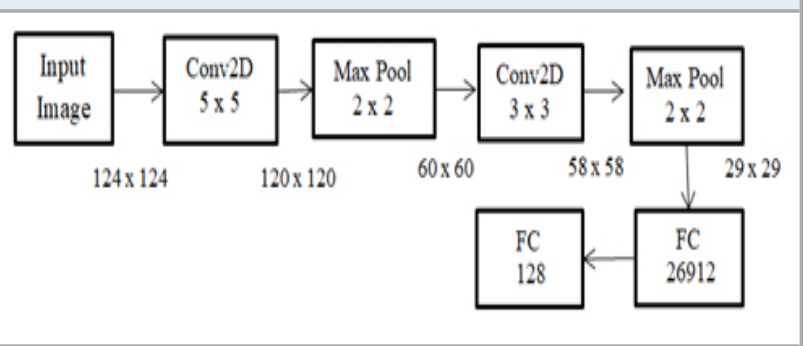

The convolution kernels are utilized over and over in each fields of the entire region, and the convolution result comprises a feature map of the input image. The convolution layer containing the weight matrix $\mathrm{w}$ and bias b. in this chapter, the size of the convolution kernel use as $5 \times 5$ and $3 \times 3$. The input size to be used as $124 \mathrm{x}$ $124 \times 3$. In this paper, we have used $2 \mathrm{D}$ convolutional network with 32 kernels of size $5 \times 5$ each which is produced an output image of size 120 x 120 .

It has used ReLU activation function which is consider to be most popular activation function. Next, The MaxPool layer is used for dimension reduction purpose with dimension as $2 \times 2$. It reduces the dimension of Conv2D network to $60 \times 60$. Again, the process of Conv2D and MaxPool layer is repeated but only change in the size of kernel. This time kernel size to be consider as $3 \times 3$. The output of MaxPool layer is being given to the Fully connected layer which flatten it to 26912. Another Fully connected layer is introduced with size as 128 . It act as a classifier for classification task. In this paper, the Stride 
of 1 is used for Con2D layer and stride of 2 is consider for MaxPool Layer with padding 0 for all the layers.

Figure 3: Sample face images under various face databases: (a)AR (b)LFWCrop (c)UK Face images (d)LAG (e)DFW (f) PSD

1. http://www2.ece.ohio-state.edu/ aleix/ARdatabase. html

2 http://conradsanderson.id.au/lfwcrop/

$3 \mathrm{http}: / /$ iab-rubric.org/resources/dfw.html

$4 \mathrm{http}: / / w w w . i v l . d i s c o . u n i m i b . i t / a c t i v i t i e s / l a r g e-a g e-g a p-$ face-verification/

5 https://cswww.essex.ac.uk/mv/allfaces/index.html 6 http://www.iab-rubric.org/resources.html

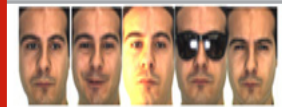

(a)

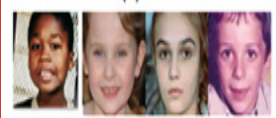

(d)

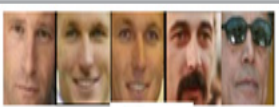

(b)

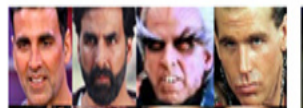

(e)

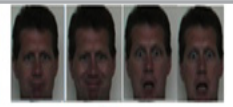

(c)

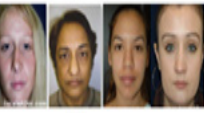

(f)
Table 1. The accuracy of proposed CNN model on various optimizer

\begin{tabular}{|c|c|c|c|c|c|c|c|c||}
\hline \multirow{2}{*}{$\begin{array}{c}\text { Types of } \\
\text { Optimizer }\end{array}$} & \multicolumn{5}{|c|}{ Face Databases } & \multirow{2}{*}{$\begin{array}{c}\text { No. of } \\
\text { epoch }\end{array}$} \\
\cline { 2 - 9 } & AR & LFW & LAG & Disguise & $\begin{array}{c}\text { UK Face } \\
\text { Database }\end{array}$ & PSD & ASPS & \\
\hline Adam & 98.4 & 98 & 97.2 & 98.5 & 97.1 & 92.2 & 94.7 & 10 \\
\hline RMSprop & 92.1 & 94 & 93.4 & 94.9 & 93.2 & 89.5 & 90.2 & 10 \\
\hline AddSign & 57.1 & 59.7 & 58.2 & 59.2 & 59.2 & 58 & 55.7 & 10 \\
\hline PowerSign & 52.7 & 53.7 & 52.2 & 51.6 & 50.2 & 51 & 50.7 & 10 \\
\hline
\end{tabular}

Figure 4: Graphical representation of results

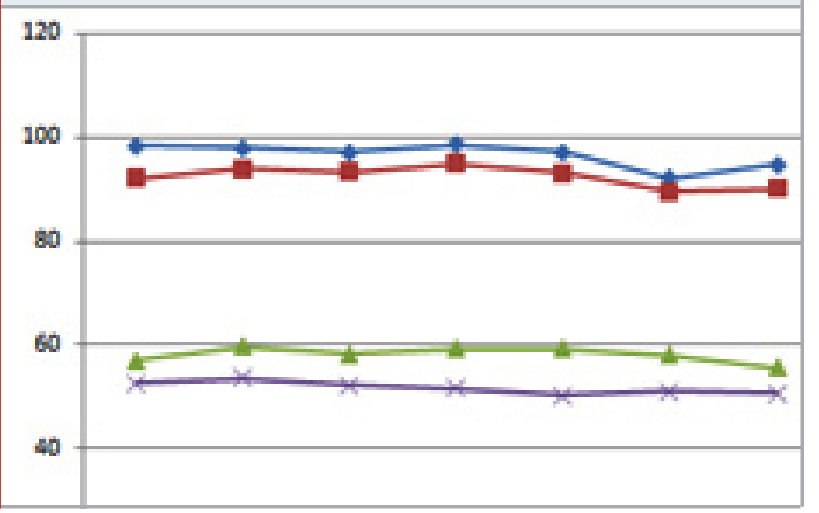

\section{RESULTS AND DISCUSSION}

We have investigated and evaluated the performance of face recognition algorithm using 7-layer CNN architecture on various face database such as AR,LFW,LAG, DFW, UKFace Database, PSD, ASPS. AR Face database1, LFWCrop face database2, DFW face image3 contains variety of face images corresponding frontal view faces with variation in facial expressions, illumination , and occlusions (scarf, sun glasses, mask). Large Age-Gap (LAG) dataset4 and UKFace Database 5 containing facial images under various age group(child to adult or young to old). The PSD6 and ASPS(American Society of Plastic Surgeon Face Database, 2019) contains various types of local plastic surgical faces before and after surgery out of which we have performed experimentation on proposed CNN model with three types of surgery. specifically, Rhinoplasty, Blepharoplasty, Lip-augmentation. The sample face images of aforementioned face database is shows in Figure 3.

The proposed proposed CNN model has binary crossentropy loss function which was shown the sum of all losses and tested this algorithm with various optimizer algorithm as PowerSign, AddSign, RMSprop, Adam. The accuracy of proposed approach is measured on the above mentioned face databases by applying types of optimizer is result is tabulated in Table II. The graphical representation of result is figured out in figure4. From the above discussion, it has been observed that the performance of proposed algorithm on PowerSign and AddSign is at lower accuracy(Kingma, D. P., \& Ba, 2015). Whereas, RMSprop gives better accuracy as compare to earlier two optimizer. Adam outperform with respect to various face databases (Bello et. al, no date).

\section{CONCLUSION}

Now a days, one of the highly demanding field in the area of image processing is face recognition. Looking to the current scenario due to COVID-19 disaster whole universe is approaching towards touch less Gadgets where less human interaction is required. Face recognition system is now important for recognition purpose in Educational institute, offices, child care unit, crime controlling bodies and where security is the major concern. In this paper, author has measure the performance of face recognition gets affected due to different factors such as aging, pose variation, facial expression, occlusion, illumination variation problem and facial plastic surgery. This makes face recognition still a most challenging task.

As per the literature, various state-of-art face recognition algorithms used to extract hand crafted facial features. This motivates us to propose the CNN model and applied on various face databases by measuring the behaviour of various optimizer. As per the literature PowerSign and AddSign perform better in terms of sequential data but when it comes to 2D face images its performance gets decrease up to 50\%. RMSprop achieved better accuracy but still struggle with finding local minimum. On a contrary, CNN outperformed with respect to Adam 
optimizer. It gives good result up to $98 \%$ for variety of face images includes, variation in expression, change of angle in terms of pose variation, change in illumination, occluded faces. However, Plastic surgery face images the performance reduces to $90 \%$. So, we can conclude that Plastic surgery face image is considered to be a more challenging task.

\section{REFERENCES}

H C Kim , D Kim, S. Y. B. (2002) 'Face recognition using LDA mixture model. Pattern Recognition', in 16th International Conference on. IEEE, pp. 486-489.

H Yu, J. Y. (2001) 'A direct LDA algorithm for highdimensional data with application to face recognition.', Pattern recognition, 34(10), pp. 2067-2070.

American Society of Plastic Surgeon Face Database (2019).

Bello et. al (no date) "Neural Optimizer Search with Reinforcement Learning”.

Boureau, Y.-L.; Ponce, J.; LeCun, Y. (2010) 'A theoretical analysis of feature pooling in visual recognition', in In Proceedings of the 27th International Conference on Machine Learning, Haifa, Israel, pp. 111-118.

D Xiaoqian , H Huan, W. A. H. (2010) 'Comparative Study of Several Face Recognition Algorithms Based on PCA', The Third International Symposium Computer Science and Computational Technology (ISCSCT 2010), p. 443.

Fredj, Hana \&t Bouguezzi, Safa \&t Souani, C. (2020) 'Face recognition in unconstrained environment with CNN', The Visual Computer Springer-Verlag GmbH Germany, part of Springer Nature.

Khadatkar, A., Khedgaonkar, R. and Patnaik, K. S. (2016) 'Occlusion invariant face recognition system', in IEEE WCTFTR 2016 - Proceedings of 2016 World Conference on Futuristic Trends in Research and Innovation for Social Welfare. doi: 10.1109/STARTUP.2016.7583985.

Kingma, D. P., \& Ba, J. L. (2015) 'Adam: a Method for Stochastic Optimization', in International Conference on Learning Representations, pp. 1-13.

L Lei, D H Kim, W J Par, et al. (2016) 'Face recognition using LBP Eigenfaces', IEICE TRANSACTIONS on Information and Systems, 97(7), pp. 1930-1932.

R.Singh (2010) Plastic Surgery Face Database Indraprastha Institute of Information Technology Delhi.

Raghuwanshi, M. M. and Singh, K. R. (2009) 'Face Recognition with Rough-Neural Network: A Rule Based Approach', International Workshop on Machine Intelligence Research, pp. 123-129.

Ridha Ilyas, Bendjillali \&t Beladgham, Moh \& Merit, Khaled \&t Miloud, K. (2020) 'Enhanced Face Recognition System Based on Deep CNN', NeuroImage IEEE, pp. 1-6.

Singh, K. R., Khedgaonkar, R. S. and Gawande, S. P. (2011) 'A New Approach to Local Plastic Surgery Face Recognition Using Near Sets', pp. 71-75. 